\title{
KINETIC STRUCTURE SIMULATIONS OF NEMATIC POLYMERS IN PLANE COUETTE CELLS. I: THE ALGORITHM AND BENCHMARKS*
}

\author{
RUHAI ZHOU ${ }^{\dagger}$, M. GREGORY FOREST ${ }^{\dagger}$, AND QI WANG ${ }^{\ddagger}$
}

\begin{abstract}
The Doi-Hess theory coupled with an anisotropic Marrucci-Greco distortional elasticity potential provides a kinetic, mean field description of the coupling between hydrodynamics, molecular orientation by excluded volume, and elastic distortions of flowing nematic liquid crystalline polymers (LCPs). In this paper we provide the first numerical algorithm and implementation of kinetic-scale models for structure formation in confined, planar Couette cells. The model and algorithm extend kinetic simulations of Larson and Ottinger [Macromolecules, 24 (1991), pp. 6270-6282], Faraoni et al. [J. Rheol., 43 (1999), pp. 829-843], Grosso et al. [Phys. Rev. Lett., 86 (2001), pp. 3184-3187], and Forest et al. [Rheol. Acta, 43 (2004), pp. 17-37; Rheol. Acta, 44 (2004), pp. 80-93] for homogeneous nematic polymers in imposed shear flows. The focus here is one-dimensional flow and LCP structures that form between oppositely moving, parallel plates, a classical model problem that has been studied in detail with continuum models [P. G. de Gennes and J. Prost, The Physics of Liquid Crystals, Clarendon Press, Oxford, 1993; G. Marrucci, Macromolecules, 24 (1991), pp. 4176-4182; G. Marrucci and F. Greco, Flow behavior of liquid crystalline polymers, in Advances in Chemical Physics, Vol. 86, Wiley, New York, pp. 331-404] and a variety of mesoscopic, second-moment orientation tensor models [T. Tsuji and A. D. Rey, J. Non-Newt. Fluid Mech., 73 (1997), pp. 127-152; R. Kupfermann, M. Kawaguchi, and M. M. Denn, J. Non-Newt. Fluid Mech., 91 (2000), pp. 255-271; G. Sgalari, G. L. Leal, and J. Feng, J. Non-Newt. Fluid Mech., 102 (2002), pp. 361-382; D. Grecov and A. D. Rey, Phys. Rev. E, 68 (2003), 061704; M. G. Forest et al., J. Rheol., 48 (2004), pp. 175-192; Z. Cui, M. G. Forest, and Q. Wang, On weak plane Couette and Poiseuille flows of nematic polymers, SIAM J. Appl. Math., submitted]. The model consists of a Smoluchowski equation for the space-time evolution of the orientational probability distribution function, coupled with a momentum flow balance equation, a constitutive equation for the extra stress, and the continuity equation. The Smoluchowski equation is first reduced to a finite set of partial differential equations in time and space for spherical harmonic amplitudes. Then we discretize the spatial variables (by the method of lines) using high-order finite differences, which reduces the full system to a large set of ordinary differential equations. Adaptive grid generation techniques are implemented. To provide an accurate and stable time integration, we employ the newly developed spectral deferred correction algorithm. We close with an application of the kinetic theory and numerical code to explore steady state flow-molecular structures in slow planar Couette experiments (low Deborah number) and low Ericksen number, where distortional elasticity dominates short-range excluded volume effects. We confirm recent analytical results based on mesoscopic closure models derived from this kinetic model, both with equal [M. G. Forest et al., J. Rheol., 48 (2004), pp. 175192] and distinct [Z. Cui, M. G. Forest, and Q. Wang, On weak plane Couette and Poiseuille flows of nematic polymers, SIAM J. Appl. Math., submitted] Frank elasticity constants, in the dual limit of low Deborah and low Ericksen numbers.
\end{abstract}

Key words. structure formation, nematic polymers, numerical simulation, kinetic model, weak shear flow

AMS subject classifications. 65N06, 65N40, 76M20

DOI. $10.1137 / 04060946 \mathrm{X}$

*Received by the editors June 4, 2004; accepted for publication (in revised form) September 23, 2004; published electronically March 25, 2005. This work was sponsored by the Air Force Office of Scientific Research, Air Force Materials Command, USAF, under grants F49620-02, 1-0086, and 60088, and the National Science Foundation through grants DMI-0115445, DMS-0204243, and DMS0308019. This work was supported in part by the NASA University Research, Engineering and Technology Institute on Bio Inspired Materials (BIMat) under award NCC-1-02037.

http://www.siam.org/journals/mms/3-4/60946.html

${ }^{\dagger}$ Department of Mathematics, University of North Carolina at Chapel Hill, Chapel Hill, NC 27599 (ruhai@amath.unc.edu, forest@amath.unc.edu).

${ }^{\ddagger}$ Department of Mathematical Sciences, Florida State University, Tallahassee, FL 32306 (wang@ math.fsu.edu). 
1. Introduction. The Doi-Hess kinetic theory with a Marrucci-Greco distortional elasticity potential provides the most resolved model for spatio-temporal structures created in plane shear flow of nematic liquid crystalline polymers (LCPs). The model reduces, by appropriate closure rules, to the wide class of mesoscopic secondmoment tensor models. The rationale for undertaking the onerous challenge of a kinetic simulation of structure formation in sheared nematic polymers is provided by recent detailed solution $[5,16,9,10]$ of the longwave limit of the planar Couette cell, extending the seminal contributions of Larson and Ottinger [20]. In this limit, the flow is imposed as simple shear (suppressing hydrodynamic feedback), distortional (Frank) elasticity and molecular anchoring are suppressed, and one studies the homogeneous, bulk response of nematic polymer monodomain responses versus shear rate and LCP concentration. This phase diagram is exceedingly complex, dominated by limit cycles and phase transitions, and sensitive to any attempt at a second-moment closure approximation (cf. [6]). Even independent of closure issues, the dynamics of orbits at fixed parameter sets is sensitive, often laden with multiple and potentially chaotic attractors [5, 16, 9, 10, 12]. To ensure accurate evolution, a high-order accurate and stable time stepper is indicated. To underscore the closure problem, 65 spherical harmonics are required to get a resolved phase diagram of all attracting states and transitions, whereas any second-moment closure is a Galerkin projection onto the first five harmonics.

Yet this longwave limit governs the genesis of structure at startup in plane Couette experiments with homogeneous initial samples. The short time interior responses in experiments and in numerical simulations consist of a linear shear profile imposed by the plates and of a nematic mesophase response to the bulk shear flow. Thus, we are compelled to develop far greater than second-moment resolution of the orientational distribution function just to get accurate early transients and to maintain this resolution as heterogeneity develops due to the incompatibility of the plate anchoring conditions and the bulk shear response of the nematic liquid. Likewise, spatial distortions of the orientational distribution generate heterogeneous viscoelastic stresses, which generate flow distortions. This feedback mechanism is a hallmark of complex fluids and soft matter $[1,24]$ and is one of the key features incorporated in the theoretical model, numerical code, and simulations of this paper.

In the present LCP-solvent example, the technological applications for ultrahigh modulus, conductivity, and barrier properties give further reasons to understand processing-induced morphology [27]. These heterogeneous molecular distributions and associated stored elastic stresses are responsible for material performance properties, yet even today "very little is known and even less is understood" [3].

Our benchmark simulations for this paper focus on structure correlations between the velocity and the features of the molecular distribution. Asymptotic analysis of mesoscopic models in the slow plate limit of this idealized Couette experiment $[2,11]$ predict two intriguing phenomena: (1) the flow profile is simple shear for sufficiently low Deborah and Ericksen numbers; and (2) the nematic polymer structures consist of a director-dominated permeation mode that spans the plate gap and plate boundary layers with order parameter distortions that are highly sensitive to plate anchoring conditions. Recent numerical studies of this mesoscopic model [15, 7, 11] predict a remarkable onset of two nonlinear flow structures as either Deborah or Ericksen number is increased beyond the asymptotically valid regime. Furthermore, these flow profiles are tunable by plate anchoring conditions. One of these flow profiles was first reported in [19], associated with a flow spurt phenomenon, for a model twodimensional nematic polymer liquid. They did not explore nontangential anchoring, 
so only one nonlinear flow structure was resolved. One of our benchmarks of the kinetic model simulation is to confirm robustness of simple shear profiles for low Deborah and Ericksen numbers and then to explore the onset of nonlinear flow structure as these parameter limits are violated. The kinetic results are compared with the mesoscopic continuation studies of $[2,11]$.

2. Governing equations. We consider nematic polymers between two plates located at $y= \pm h$, in Cartesian coordinates $\mathbf{x}=(x, y, z)$, and moving with corresponding velocity $\mathbf{v}=\left( \pm v_{0}, 0,0\right)$, respectively. There are two apparent length scales in this problem: the gap width $2 h$, an external length scale, and the finite range $l$ of molecular interaction, an internal length scale set by the distortional elasticity in the Doi-Marrucci-Greco (DMG) model. The plates move relative to each other at a constant speed, which sets a bulk flow time scale $\left(t_{v}=\frac{h}{v_{0}}\right)$; the nematic average rotary diffusivity sets another (internal) time scale $\left(t_{l c p}=\frac{1}{6 D_{r}}\right)$. We nondimensionalize the DMG model using the internal time scale, following [7]. The dimensionless Smoluchowski equation for the orientational probability distribution function (PDF) $f(\mathbf{m}, \mathbf{x}, t)$ is

$$
\begin{aligned}
& \frac{D f}{D t}=\mathcal{R} \cdot[(\mathcal{R} f+f \mathcal{R} V)]-\mathcal{R} \cdot[\mathbf{m} \times \dot{\mathbf{m}} f] \\
& \dot{\mathbf{m}}=\Omega \cdot \mathbf{m}+a[\mathbf{D} \cdot \mathbf{m}-\mathbf{D}: \mathbf{m m m}],
\end{aligned}
$$

where $D / D t$ is the material derivative, and the intermolecular potential $V$ is given by

$$
V=-\frac{3 N}{2}\left[\left(\mathbf{I}+\frac{1}{3 E r} \Delta\right) \mathbf{M}: \mathbf{m m}+\frac{\theta}{3 E r}(\mathbf{m m}:(\nabla \nabla \cdot \mathbf{M}))\right] .
$$

In the Smoluchowski equation (1), $\mathcal{R}$ is the rotational operator:

$$
\mathcal{R}=\mathbf{m} \times \frac{\partial}{\partial \mathbf{m}},
$$

and $\mathbf{D}$ and $\Omega$ are rate-of-strain and vorticity tensors, which are symmetric and antisymmetric parts of $\nabla \mathbf{v}$, respectively. The Ericksen number $\operatorname{Er}$ in (2) measures short-ranged nematic potential relative to distortional elasticity strength (see [7, 13] for details). The parameter $0 \leq \theta \leq 1$ relates to Frank elastic constants; $\theta=0$ is the isotropic case where all constants are equal, and $\theta>0$ allows for distinct constants. In the potential equation (2), $\mathbf{M}$ is the second moment tensor of the $\operatorname{PDF} f$ :

$$
\mathbf{M}=\int_{\|\mathbf{m}\|=1} \mathbf{m m} f(\mathbf{m}, \mathbf{x}, t) d \mathbf{m} .
$$

The unit eigenvector of $\mathbf{M}$ corresponding to the largest eigenvalue is called the major director, or peak, of the $\operatorname{PDF} f$. It is standard to define the orientation tensor $\mathbf{Q}$ as the traceless part of $\mathbf{M}$,

$$
\mathbf{Q}=\mathbf{M}-\frac{1}{3} \mathbf{I}
$$

The dimensionless forms of the balance of linear momentum, the stress constitu- 
tive equation, and the continuity equation are [28]

$$
\begin{aligned}
\frac{d \mathbf{v}}{d t}= & \nabla \cdot(-p \mathbf{I}+\tau), \\
\tau= & \left(2 / R e+\mu_{3}(a)\right) \mathbf{D}+a \alpha\left(\mathbf{M}-\frac{\mathbf{I}}{3}-N \mathbf{M} \cdot \mathbf{M}+N \mathbf{M}: \mathbf{M}_{4}\right) \\
& -a \frac{\alpha}{6 E r}\left(\Delta \mathbf{M} \cdot \mathbf{M}+\mathbf{M} \cdot \Delta \mathbf{M}-2 \Delta \mathbf{M}: \mathbf{M}_{4}\right) \\
& -\frac{\alpha}{12 E r}(2(\Delta \mathbf{M} \cdot \mathbf{M}-\mathbf{M} \cdot \Delta \mathbf{M})+(\nabla \mathbf{M}: \nabla \mathbf{M}-(\nabla \nabla \mathbf{M}): \mathbf{M})) \\
& -a \frac{\alpha \theta}{6 E r}\left[\mathbf{M} \cdot \mathbf{M}_{d}+\mathbf{M}_{d} \cdot \mathbf{M}-4(\nabla \nabla \cdot \mathbf{M}): \mathbf{M}_{4}\right] \\
& -\frac{\alpha \theta}{6 E r}\left[\mathbf{M}_{d} \cdot \mathbf{M}-\mathbf{M} \cdot \mathbf{M}_{d}+(\nabla \nabla \cdot \mathbf{M}) \cdot \mathbf{M}-\mathbf{M}_{\beta j, \alpha} \mathbf{M}_{i j, i}\right] \\
& +\left[\mu_{1}(a)(\mathbf{D} \cdot \mathbf{M}+\mathbf{M} \cdot \mathbf{D})+\mu_{2}(a) \mathbf{D}: \mathbf{M}_{4}\right], \\
\nabla \cdot \mathbf{v}= & 0
\end{aligned}
$$

where

$$
\mathbf{M}_{d}=\nabla \nabla \cdot \mathbf{M}+(\nabla \nabla \cdot \mathbf{M})^{T}, \quad \mathbf{M}_{4}=\int_{\|\mathbf{m}\|=1} \operatorname{mmmm} f(\mathbf{m}, \mathbf{x}, t) d \mathbf{m}
$$

where $\mathbf{M}_{4}$ is clearly the fourth-moment tensor of the PDF, while $\mathbf{M}_{d}$ is the symmetric part of the tensor of second-order spatial derivatives of $\mathbf{M}$, with explicit properties given in (19) and (20) below. In the stress formula (6), $\mu_{1}, \mu_{2}, \mu_{3}$ are three nematic Reynolds numbers, $\alpha$ measures the strength of elastic energy relative to kinetic energy, $N$ is the nematic concentration, and $-1 \leq a \leq 1$ is the spheroidal molecule shape parameter, $a=\left(r^{2}-1\right) /\left(r^{2}+1\right)$, where $r$ is the molecule aspect ratio.

The plate boundary conditions on the dimensionless velocity $\mathbf{v}$ are given by

$$
\left.\mathbf{v}\right|_{y= \pm 1}=( \pm D e, 0,0)
$$

where the Deborah number De measures the bulk flow velocity relative to the internal nematic relaxation rate (see [7, 13]). Following previous studies [3, 19, 11], we assume homogeneous anchoring at the plates, given by the quiescent nematic equilibrium $f_{e}$,

$$
f(\mathbf{m}, y= \pm 1, t)=f_{e}(\mathbf{m}) .
$$

Note that the quiescent nematic equilibrium has $\mathrm{O}(3)$ symmetry [8]; that is, any rotation of a quiescent nematic equilibrium $f_{e}$ is also a solution, which is then broken by a plate anchoring condition. We discuss below how to impose the boundary conditions in numerical simulations.

3. Numerical method. Each of the three independent variables in the model system requires a separate numerical strategy: the orientation variable $\mathbf{m} \in S^{2}$, the spatial variable $\mathbf{x}$, and the time variable $t$. Each variable dependence is sufficiently sensitive such that special numerical methods are required. For this paper we restrict ourselves to one space dimension. We first expand the PDF using spherical harmonic functions (the approach adopted in $[20,5]$ for homogeneous monodomains in imposed shear flows), reducing the Smoluchowski equation to a system of PDEs in the spatial variable and time. Then we use the method of lines to derive a system of ODEs by discretizing the spatial variable using fourth-order finite difference methods. Finally, we perform time integration using a preconditioned Euler method coupled with spectral deferred corrections [4] to get a fourth-order method. Adaptive time stepping and a moving spatial grid technique are used.

Note that the treatment of the balance equation in (6) is straightforward, so we describe only the numerical procedure for the Smoluchowski equation (1). 
3.1. Spherical harmonic expansion. Spherical harmonic functions are defined by

$$
Y_{l}^{m}(\mathbf{m})=P_{l}^{m}(\cos \gamma) e^{i m \psi}, \quad l=0,1,2, \ldots, \quad m=-l, \ldots, 0, \ldots, l,
$$

where $P_{l}^{m}$ are the associated Legendre polynomials, and $\gamma, \psi$ are spherical coordinates with

$$
\mathbf{m}=(\sin \gamma \cos \psi, \sin \gamma \sin \psi, \cos \gamma) .
$$

Note that spherical harmonic functions defined here have been normalized so that they form an orthonormal basis on the unit sphere.

The PDF as modeled in the Smoluchowski equation is reflection symmetric, that is, $f(-\mathbf{m})=f(\mathbf{m})$, and the integration over the unit sphere is unity. Therefore, $f$ admits the following expansion:

$$
f=\frac{1}{2 \sqrt{\pi}} Y_{0}^{0}(\mathbf{m})+\sum_{l>0}^{\text {even }} \sum_{m=-l}^{l} a_{l}^{m}(\mathbf{x}, t) Y_{l}^{m}(\mathbf{m}),
$$

where $Y_{0}^{0}(\mathbf{m})=\frac{1}{2 \sqrt{\pi}}$. Under this expansion, we now briefly show how to derive expansions for each term in the Smoluchowski equation.

Using the spectral property of the rotational operator,

$$
\mathcal{R} \cdot \mathcal{R} Y_{l}^{m}=-l(l+1) Y_{l}^{m},
$$

we immediately have

$$
\mathcal{R} \cdot \mathcal{R} f=-\sum_{l>0}^{\text {even }} \sum_{m=-l}^{l} l(l+1) a_{l}^{m}(\mathbf{x}, t) Y_{l}^{m} .
$$

We split the potential $V$, equation (2), into three parts. First, we calculate

$$
V_{1}=\mathbf{m m}: \mathbf{M}=\frac{4 \pi}{3} Y_{0}^{0}+\frac{8 \pi}{15} \sum_{p=-2}^{2} a_{2}^{p} Y_{2}^{p}
$$

Therefore,

$$
\mathcal{R} \cdot\left(f \mathcal{R} V_{1}\right)=\mathcal{R} f \cdot \mathcal{R} V_{1}+f \mathcal{R} \cdot \mathcal{R} V_{1}
$$

$$
=-\frac{4}{5} \sum_{p=-2}^{2} a_{2}^{p} Y_{2}^{p}+\frac{8 \pi}{15} \sum_{l>0}^{\text {even }} \sum_{m=-l}^{l} \sum_{p=-2}^{2} a_{2}^{p} a_{l}^{m}\left(\mathcal{R} Y_{2}^{p} \cdot \mathcal{R} Y_{l}^{m}-6 Y_{2}^{p} Y_{l}^{m}\right) .
$$

The dot products $\mathcal{R} Y_{2}^{p} \cdot \mathcal{R} Y_{l}^{m}$ can be represented as a sum of products of two spherical harmonics:

$$
\begin{aligned}
\mathcal{R} Y_{l}^{m} \cdot \mathcal{R} Y_{2}^{-2} & =-c_{l}^{-m} Y_{2}^{-1} Y_{l}^{m-1}+2 m Y_{2}^{-2} Y_{l}^{m}, \\
\mathcal{R} Y_{l}^{m} \cdot \mathcal{R} Y_{2}^{-1} & =-\sqrt{\frac{3}{2}} c_{l}^{-m} Y_{2}^{0} Y_{l}^{m-1}+m Y_{2}^{-1} Y_{l}^{m}-c_{l}^{m} Y_{2}^{-2} Y_{l}^{m+1}, \\
\mathcal{R} Y_{l}^{m} \cdot \mathcal{R} Y_{2}^{0} & =-\sqrt{\frac{3}{2}} c_{l}^{-m} Y_{2}^{1} Y_{l}^{m-1}-\sqrt{\frac{3}{2}} c_{l}^{m} Y_{2}^{-1} Y_{l}^{m+1}, \\
\mathcal{R} Y_{l}^{m} \cdot \mathcal{R} Y_{2}^{1} & =-\sqrt{\frac{3}{2}} c_{l}^{m} Y_{2}^{0} Y_{l}^{m+1}-m Y_{2}^{1} Y_{l}^{m}-c_{l}^{-m} Y_{2}^{2} Y_{l}^{m-1}, \\
\mathcal{R} Y_{l}^{m} \cdot \mathcal{R} Y_{2}^{2} & =-c_{l}^{m} Y_{2}^{1} Y_{l}^{m+1}-2 m Y_{2}^{2} Y_{l}^{m}
\end{aligned}
$$


where

$$
c_{l}^{m}=\sqrt{(l-m)(l+m+1)} .
$$

For $V_{2}=\mathbf{m m}: \Delta \mathbf{M}$, the formula is the same as (16) with $a_{2}^{p}$ replaced with $\partial^{2} a_{2}^{p} / \partial y^{2}$.

For the other term in the potential, we have

$$
V_{3}=\mathbf{m m}:(\nabla \nabla \cdot \mathbf{M})=\sigma Y_{0}^{0}+\frac{8 \pi}{15} \sum_{p=-2}^{2} \sigma_{p} Y_{2}^{p},
$$

where

$$
\begin{aligned}
\sigma & =-\frac{2 \pi}{9 \sqrt{5}}\left(2 \frac{\partial^{2} a_{2}^{0}}{\partial y^{2}}+\sqrt{6}\left(\frac{\partial^{2} a_{2}^{2}}{\partial y^{2}}+\frac{\partial^{2} a_{2}^{-2}}{\partial y^{2}}\right)\right) \\
\sigma_{-2} & =\frac{1}{12}\left(6 \frac{\partial^{2} a_{2}^{-2}}{\partial y^{2}}+\sqrt{6} \frac{\partial^{2} a_{2}^{0}}{\partial y^{2}}\right) \\
\sigma_{-1} & =\frac{1}{4}\left(\frac{\partial^{2} a_{2}^{-1}}{\partial y^{2}}+\frac{\partial^{2} a_{2}^{1}}{\partial y^{2}}\right) \\
\sigma_{0} & =\frac{1}{12}\left(2 \frac{\partial^{2} a_{2}^{0}}{\partial y}+\sqrt{6}\left(\frac{\partial^{2} a_{2}^{-2}}{\partial y^{2}}+\frac{\partial^{2} a_{2}^{2}}{\partial y^{2}}\right)\right) \\
\sigma_{1} & =\frac{1}{4}\left(\frac{\partial^{2} a_{2}^{-1}}{\partial y^{2}}+\frac{\partial^{2} a_{2}^{1}}{\partial y^{2}}\right) \\
\sigma_{2} & =\frac{1}{12}\left(6 \frac{\partial^{2} a_{2}^{2}}{\partial y^{2}}+\sqrt{6} \frac{\partial^{2} a_{2}^{0}}{\partial y^{2}}\right) .
\end{aligned}
$$

Again, we get a similar formula to (16) that is omitted here.

The last term in (1) is the shear flow contribution, which can be simplified as

$$
\mathcal{R} \cdot[\mathbf{m} \times \dot{\mathbf{m}} f]=\frac{\partial v_{x}}{\partial y} \cdot G(f),
$$

where $v_{x}$ is the $x$-component of $\mathbf{v}$, and

$$
\begin{aligned}
G(f)= & a \sqrt{\frac{8 \pi}{15}}\left(\frac{1}{2}\left(Y_{2}^{1}-Y_{2}^{-1}\right) \mathcal{R}_{x} f+\frac{1}{2} i\left(Y_{2}^{1}+Y_{2}^{-1}\right) \mathcal{R}_{y} f+\left(Y_{2}^{2}+Y_{2}^{-2}\right) \mathcal{R}_{z} f\right) \\
& -\mathcal{R}_{z} f+3 \sqrt{\frac{8 \pi}{15}} i a\left(Y_{2}^{2}-Y_{2}^{-2}\right) f,
\end{aligned}
$$

where $\mathcal{R}_{x}, \mathcal{R}_{y}$, and $\mathcal{R}_{z}$ are three components of the operator $\mathcal{R}$ in Cartesian coordinates, and $i=\sqrt{-1}$. $G$ is a linear operator with the following action on spherical harmonics:

$$
i G\left(Y_{l}^{m}\right)=m Y_{l}^{m}+a\left(\sum_{p=-2}^{2} \alpha_{l, m, p} Y_{l+p}^{m-2}-\sum_{p=-2}^{2} \alpha_{l,-m, p} Y_{l+p}^{m+2}\right)
$$

with the coefficients determined by

$$
\begin{aligned}
\alpha_{l, m,-2} & =-\frac{(l-2) \sqrt{(-3+l+m)(-2+l+m)(-1+l+m)(l+m)}}{2 \sqrt{(-3+2 l)(1+2 l)}(-1+2 l)} \\
\alpha_{l, m, 0} & =-\frac{3 \sqrt{(1+l-m)(2+l-m)(-1+l+m)(l+m)}}{2(-1+2 l)(3+2 l)} \\
\alpha_{l, m, 2} & =\frac{(3+l) \sqrt{(1+l-m)(2+l-m)(3+l-m)(4+l-m)}}{2 \sqrt{(1+2 l)(5+2 l)}(3+2 l)} \\
\alpha_{l, m, p} & =0 \quad \text { if } p \neq-2,0,2 .
\end{aligned}
$$


TABLE 1

Constants that appear in (25).

\begin{tabular}{|c|c|c|c|}
\hline \hline$p$ & $b_{l-2}^{p, m}$ & $b_{l}^{p, m}$ & $b_{l+2}^{p, m}$ \\
\hline-2 & $\frac{\sqrt{(l+m)(l+m-1)(l+m-2)(l+m-3)}}{4(2 l-1) \sqrt{(2 l-3)(2 l+1)}}$ & $-\frac{\sqrt{(l+m)\left[l^{2}-(m-1)^{2}\right](l-m+2)}}{2(2 l-1)(2 l+3)}$ & $\frac{\sqrt{(l-m+1)(l-m+2)(l-m+3)(l-m+4)}}{4(2 l+3) \sqrt{(2 l+1)(2 l+5)}}$ \\
\hline-1 & $-\frac{\sqrt{\left(l^{2}-m^{2}\right)(l+m-1)(l+m-2)}}{2(2 l-1) \sqrt{(2 l-3)(2 l+1)}}$ & $\frac{(2 m-1) \sqrt{(l+m)(l-m+1)}}{2(2 l-1)(2 l+3)}$ & $\frac{\sqrt{\left[(l+1)^{2}-m^{2}\right](l-m+2)(l-m+3)}}{2(2 l+3) \sqrt{(2 l+1)(2 l+5)}}$ \\
\hline 0 & $\frac{3 \sqrt{\left[(l-1)^{2}-m^{2}\right]\left(l^{2}-m^{2}\right)}}{2(2 l-1) \sqrt{6(2 l-3)(2 l+1)}}$ & $\frac{l^{2}-3 m^{2}+1}{\sqrt{6}(2 l-1)(2 l+3)}$ & $\frac{3 \sqrt{\left[(l+2)^{2}-m^{2}\right]\left[(l+1)^{2}-m^{2}\right]}}{2(2 l+3) \sqrt{6(2 l+1)(2 l+5)}}$ \\
\hline 1 & $-\frac{\sqrt{\left(l^{2}-m^{2}\right)(l-m-1)(l-m-2)}}{2(2 l-1) \sqrt{(2 l-3)(2 l+1)}}$ & $\frac{(2 m+1) \sqrt{(l-m)(l+m+1)}}{2(2 l-1)(2 l+3)}$ & $\frac{\sqrt{\left[(l+1)^{2}-m^{2}\right](l+m+2)(l+m+3)}}{2(2 l+3) \sqrt{(2 l+1)(2 l+5)}}$ \\
\hline 2 & $\frac{\sqrt{(l-m)(l-m-1)(l-m-2)(l-m-3)}}{4(2 l-1) \sqrt{(2 l-3)(2 l+1)}}$ & $-\frac{\sqrt{(l-m)\left[l^{2}-(m+1)^{2}\right](l+m+2)}}{2(2 l-1)(2 l+3)}$ & $\frac{\sqrt{(l+m+1)(l+m+2)(l+m+3)(l+m+4)}}{4(2 l+3) \sqrt{(2 l+1)(2 l+5)}}$ \\
\hline \hline
\end{tabular}

Next we multiply the Smoluchowski equation by $\left(Y_{l}^{m}\right)^{*}$, the complex conjugate of $Y_{l}^{m}$, and integrate over the unit sphere. During this process, we need to calculate integrals of products of three spherical harmonic functions. The "3j-symbol" (see, e.g., [23]) can be used; instead, because one spherical harmonic function in the product is of second order, we use the following expansion to deal with products appearing in (16) and (17):

$$
Y_{2}^{p} Y_{l}^{m}=\sqrt{\frac{15}{2 \pi}}\left(b_{l-2}^{p, m} Y_{l-2}^{m+p}+b_{l}^{p, m} Y_{l}^{m+p}+b_{l+2}^{p, m} Y_{l+2}^{m+p}\right), \quad p=-2,-1,0,1,2,
$$

where $b_{l-2}^{p, m}, b_{l}^{p, m}$, and $b_{l+2}^{p, m}$ are constants listed in Table 1. Using the orthonormality of spherical harmonic functions, we then get a system of PDEs for all amplitudes $a_{l}^{m}(\mathbf{x}, t)$. In our numerical computations, the system is truncated so that the maximum $l$ is 10 . In this case, 65 PDEs are solved for the orientation field, which are of nonlinear, reaction-diffusion type.

3.2. Spatial discretization and grid generation. For spatial discretization, we use a standard fourth-order central finite difference method at central grid points and a lopsided fourth-order finite difference method at the grid points near the boundary. Using the method of lines, we get a system of ODEs which will be solved in the next subsection.

Because there are sharp gradients in the solution of the Smoluchowski equation, an adaptive grid is introduced. The grid adaptation scheme can be found in, e.g., [18]. In this paper, we neglect the variations in the flow direction $x$ and the vorticity direction $z$. Only the structure along the flow-gradient direction $y$ is considered. In this one-dimensional case, the variational approach for grid adaptation in [18] is equivalent to several other simple methods. One of these is the notion of the equidistribution of a monitor function that we use here. In the equidistribution approach one first creates a positive monitor function $M(y)$ and then determines a mesh $-1=y_{0}<y_{1}<\cdots<y_{N-1}<y_{N}=1$ such that the average of $M$ has the same constant value over each subinterval $\left[y_{k}, y_{k+1}\right]$; that is,

$$
\int_{y_{k}}^{y_{k+1}} M(y) d y=C, \quad k=0,1, \ldots, N-1
$$


where the constant $C$ is

$$
C=\frac{1}{N} \int_{-1}^{1} M(y) d y .
$$

In practice, $M$ is only available at the old grid points, so piecewise linear interpolation is used to compute intermediate values. To produce the adapted grid points we use Newton iteration to solve (26) for $y_{k+1}$ given $y_{k}$. That is, we use the iteration

$$
s^{m+1}=s^{m}-\frac{1}{M\left(s^{m}\right)}\left[\int_{y_{k}}^{s^{m}} M(y) d y-C\right]
$$

and then let $y_{k+1}=s^{\infty}$. Because $M$ is piecewise linear, only a few iterations are needed to meet our error tolerance.

We choose the initial grid as uniform. To avoid dramatic changes in the grid, we iterate the grid generation algorithm several times before the time integration begins. During the time integration, the grid is updated at every step. Underrelaxation of the grid generation is also used to avoid instabilities; that is, the generated grid is given by

$$
y_{k}=y_{k}^{\text {old }}+\alpha\left(y_{k}^{\text {new }}-y_{k}^{\text {old }}\right),
$$

with $0<\alpha<1$. We usually use $\alpha=0.75$.

To track sharp spatial gradients during structure formation, we define the monitor function $M$ from simple combinations of the first and second derivatives of the solution:

$$
M(y)=1+C_{1}\left(\frac{\left|T_{y}\right|}{\max \left|T_{y}\right|}\right)+C_{2}\left(\frac{\left|T_{y y}\right|}{\max \left|T_{y y}\right|}\right),
$$

where $C_{1}$ and $C_{2}$ are two positive constants, and $T$ is a solution component or a combination of several solution components. Grid adaptive schemes using such monitor functions are called feature adaptive because they adapt the grid to some features of the solution, which can reduce the error in the solution.

3.3. Spectral deferred corrections. From the method of lines, after spatial discretization, we get a system of ODEs which we write in the following form:

$$
\begin{aligned}
U^{\prime}(t) & =F(U(t)), \\
U(a) & =U_{a} .
\end{aligned}
$$

The method of spectral deferred correction is used to solve ODEs in the equivalent integral equation form:

$$
U(t)=U_{a}+\int_{a}^{t} F(U(s)) d s .
$$

Assume that the solution has been numerically computed to high order at $t=t_{0}$ and our goal is to get a high-order solution at $t=t_{s}$. We divide the interval $\left[t_{0}, t_{s}\right]$ using $m$ quadrature nodes $t_{1}<t_{2}<\cdots<t_{m}$ with $h_{j}=t_{j+1}-t_{j}$ and $h=t_{s}-t_{0}$. We allow $t_{0} \leq t_{1}$ and $t_{m} \leq t_{s}$ so that we can use Gauss quadrature. Let $U_{0}$ be the high-order solution at $t_{0}$, and let $\tilde{U}_{1}^{0}, \tilde{U}_{2}^{0}, \ldots, \tilde{U}_{m}^{0}$ be the first-order approximation 
of the solutions at quadrature nodes, which is computed by a preconditioned Euler method (with $\left.\tilde{U}_{0}^{0}=U_{0}\right)$ :

$$
\tilde{U}_{j+1}^{0}=\tilde{U}_{j}^{0}+\left(I-h_{j} J_{j}\right)^{-1} h_{j} F\left(\tilde{U}_{j}^{0}\right), \quad j=0,1, \ldots, m-1,
$$

where $J_{j}$ is the approximation of the Jacobian of $F$ which we choose as block tridiagonal.

The Lagrange interpolant $\tilde{U}^{0}$ of the first-order solution can be determined by

$$
\tilde{U}^{0}(t)=\sum_{j=1}^{m} c_{j}(t) \tilde{U}_{j}^{0}, \quad \text { with } \quad c_{j}(t)=\prod_{i \neq j} \frac{t-t_{i}}{t_{j}-t_{i}},
$$

from which we can calculate the residue for the Lagrange interpolant from the integral equation (31):

$$
\epsilon^{0}(t)=U\left(t_{0}\right)+\int_{t_{0}}^{t} F\left(\tilde{U}^{0}(s)\right) d s-\tilde{U}^{0}(t), \quad t_{0} \leq t \leq t_{s}
$$

With this residue, we construct the neighboring equation for (31):

$$
U(t)=U\left(t_{0}\right)+\int_{t_{0}}^{t} F(U(s)) d s-\epsilon^{0}(t)
$$

which has the Lagrange interpolant $\tilde{U}^{0}(t)$, equation (33), as its exact solution. Although the exact solution is known, we still use the first-order preconditioned Euler method, as described above, to find it numerically. Denote the numerical solution as $\bar{U}_{j}^{0}$. Then (with $\bar{U}_{0}^{0}=U_{0}$ )

$$
\bar{U}_{j+1}^{0}=\bar{U}_{j}^{0}+\left(I-h_{j} J_{j}\right)^{-1}\left(h_{j} F\left(\bar{U}_{j}^{0}\right)-\left(\epsilon_{j+1}^{0}-\epsilon_{j}^{0}\right)\right), \quad j=0, \ldots, m-1 .
$$

The correction

$$
\tilde{U}_{j}^{1}=\tilde{U}_{j}^{0}+\left(\tilde{U}_{j}^{0}-\bar{U}_{j}^{0}\right), \quad j=0, \ldots, m-1,
$$

then provides second-order solutions. To produce third-order solutions, we construct another neighboring problem as in (34) and (35) with $\tilde{U}^{0}$ replaced by $\tilde{U}^{1}$ and $\epsilon^{0}$ replaced by $\epsilon^{1}$, and solve this new neighboring problem using (36) with $\epsilon^{0}$ replaced by $\epsilon^{1}$ and $\bar{U}^{0}$ replaced by $\bar{U}^{1}$. After that, the third-order solution is given by

$$
\tilde{U}_{j}^{2}=\tilde{U}_{j}^{0}+\left(\tilde{U}_{j}^{1}-\bar{U}_{j}^{1}\right), \quad j=0, \ldots, m-1 .
$$

This idea of deferred corrections is first used in [14] and the references therein, where it is applied to the differential equations (30) directly. In this work, we use the integral form of the differential equations as suggested in [4]. As in [4], we call the method spectral deferred corrections, especially when Gauss quadrature nodes are used. The following is the outline of the spectral deferred correction method:

- Compute first-order approximations at the quadrature nodes with $\tilde{U}_{0}^{0}=U_{0}$ :

$$
\tilde{U}_{j+1}^{0}=\tilde{U}_{j}^{0}+\left(I-h_{j} J_{j}\right)^{-1} h_{j} F\left(\tilde{U}_{j}^{0}\right), \quad j=0,1, \ldots, m-1 .
$$

- Make $k_{\max }$ corrections: 
For each $k=0,1, \ldots, k_{\max }-1$, set $\bar{U}_{0}^{k}=U_{0}$ and compute the following:

For $j=0, \ldots, m-1$

$$
\begin{aligned}
& \epsilon_{j+1}^{k}-\epsilon_{j}^{k}=\int_{t_{j}}^{t_{j+1}} F\left(\tilde{U}^{k}(s)\right) d s-\left(\tilde{U}_{j+1}^{k}-\tilde{U}_{j}^{k}\right), \\
& \bar{U}_{j+1}^{k}=\bar{U}_{j}^{k}+\left(I-h_{j} J_{j}\right)^{-1}\left(h_{j} F\left(\bar{U}_{j}^{k}\right)-\left(\epsilon_{j+1}^{k}-\epsilon_{j}^{k}\right)\right), \\
& \tilde{U}_{j+1}^{k+1}=\tilde{U}_{j+1}^{0}+\left(\tilde{U}_{j+1}^{k}-\bar{U}_{j+1}^{k}\right) .
\end{aligned}
$$

- Extrapolate, if necessary, the $\tilde{U}_{j}^{k_{\max }}$ values to the value $\tilde{U}_{s}$ at $t_{s}$ using a high-order method.

In our computation, we use 5 Gaussian quadrature nodes. The maximum order that the spectral deferred correction method can achieve is 10 . Usually, we do corrections until the order is 4 or 6 . The stability is enhanced if the order is increased.

3.4. Summary of the numerical method. After the spherical harmonic expansion, we get a system of PDEs for amplitudes $a_{l}^{m}(y, t), l=2,4, \ldots, L ; m=$ $-l, \ldots, l$. We define $\mathbf{u}$ as the vector which contains all $a_{l}^{m}$; then the obtained PDEs can be written in the form

$$
\mathbf{u}_{t}=g\left(\mathbf{u}, \frac{\partial \mathbf{u}}{\partial y}, \frac{\partial^{2} \mathbf{u}}{\partial y^{2}}\right)
$$

We view the grid adaption process described in subsection 3.2 as a moving grid that corresponds to a time-dependent change of coordinates of the form

$$
y=y(\xi, \tau), \quad t=\tau,
$$

where $\xi$ is in the logical space and $y$ is the physical space [18]. Under this change of coordinates,

$$
\mathbf{u}_{y}=\frac{1}{y_{\xi}} \mathbf{u}_{\xi}, \quad \mathbf{u}_{\tau}=\mathbf{u}_{t}+\frac{y_{\tau}}{y_{\xi}} \mathbf{u}_{\xi}
$$

The first formula is simply the chain rule used numerically to compute first-order spatial derivatives using the uniform grid in logical space. Second-order spatial derivatives can be similarly derived. The other formula indicates the Lagrange form of the original PDEs, which takes into account the moving grid in the physical space. Combining this equation with the grid generation (26), we are solving the coupled equations:

$$
\begin{aligned}
\mathbf{u}_{\tau} & =\mathbf{u}_{t}+\frac{y_{\tau}}{y_{\xi}} \mathbf{u}_{\xi}, \\
\int_{y_{k}}^{y_{k+1}} M(y) d y & =C, \quad k=0,1, \cdots, N-1 .
\end{aligned}
$$

At every time step, we first update the grid points using (41) and then solve PDEs (40) using methods described in the previous two subsections.

In our approach to time integration (spectral deferred corrections), there are several substeps determined by quadrature nodes in each time interval $\left[t_{0}, t_{s}\right]$. This makes it possible to calculate the time derivative of the grid points, i.e., $y_{\tau}$ simply. After the new grid is generated at $t_{s}$, we assume the grid is moving linearly in the whole time step $\left[t_{0}, t_{s}\right]$. In this way, $y_{\tau}$ is kept constant in this time interval. 
To make our algorithm more efficient, we also use adaptive time steps. The spectral deferred correction method provides an estimate of local time integration error. Suppose $\mathbf{u}_{1}$ is the $p$ th order solution and $\mathbf{u}_{2}$ is the $(p-1)$ th order solution. The local relative error $\operatorname{err}_{n+1}$ after the $n$th time step is estimated by the 2-norm of their differences. Following Gustafsson theory (see, e.g., [17]), the time step size $\Delta t_{n+1}$ at the $(n+1)$ th step is given by

$$
\Delta t_{n+1}=\alpha \Delta t_{n}\left(\frac{t o l}{(e r r)_{n+1}}\right)^{1 / p} \frac{\Delta t_{n}}{\Delta t_{n-1}}\left(\frac{(e r r)_{n}}{(e r r)_{n+1}}\right)^{1 / p},
$$

where $\alpha$ is a fudge factor, and tol is the given tolerance. This procedure is efficient for stiff problems. Alternatively, we also use the formula (see, e.g., [17])

$$
\Delta t_{n+1}=\alpha \frac{\Delta t_{n}}{(e r r)_{n+1}^{1 / p}}
$$

for nonstiff problems.

3.5. Numerical tests. We are now in position to describe the boundary conditions in detail. We assume homogeneous anchoring at the plates, given by the quiescent nematic equilibrium (9), which is $\mathrm{O}(3)$ symmetric. To select the equilibrium with major director $\mathbf{n}$ specified by the plate anchoring condition, we first construct a uniaxial traceless tensor $\mathbf{Q}_{0}$ from

$$
\mathbf{Q}_{0}=s\left(\mathbf{n n}-\frac{1}{3} \mathbf{I}\right),
$$

with suitable order parameters $s$. Then we construct $f_{0}$ with $a_{2}^{p}, p=-2,-1,0,1,2$, derived from this $\mathbf{Q}_{0}$ using the relationships between the PDF and its second-moment projection:

$$
\begin{aligned}
a_{2}^{0} & =-\frac{3}{4} \sqrt{\frac{5}{\pi}}\left(Q_{x x}+Q_{y y}\right), \\
\Re\left(a_{2}^{2}\right) & =\frac{1}{4} \sqrt{\frac{15}{2 \pi}}\left(Q_{x x}-Q_{y y}\right), \\
\Im\left(a_{2}^{2}\right) & =-\frac{1}{2} \sqrt{\frac{15}{2 \pi}} Q_{x y}, \\
a_{l}^{m} & =0 \quad \text { for all other } l \text { and } m .
\end{aligned}
$$

We set $D e=0$ (no flow), suppress the space variable $\mathbf{x}$ in the Smoluchowski equation, and numerically solve the ODE system from the starting state $f_{0}$. This orbit converges to the required equilibrium $f_{e}(\mathbf{m})$, which is guaranteed to have $\mathbf{n}$ as its major director.

We first test the order of convergence of our method. The primary parameters taken are $E r=100, D e=0.1$. The final time is 0.1. We use the numerical solution obtained with time step size $2 \times 10^{-4}$ and grid spacing 0.05 as the reference solution. We then decrease these two quantities in the fourth-order numerical method. Results are summarized in Table 2, where the absolute 2-norm error is reported. We clearly see the convergence rate is 4 as expected. Note that, in this test, the spatial grid is uniform for all runs. 
TABLE 2

Fourth-order convergence for the test problem. $h$ is the grid space; ts is the time step.

\begin{tabular}{|c||c|c|c|}
\hline \hline$h$ & 0.2 & 0.1 & 0.05 \\
\hline$t s$ & $8 . \mathrm{E}-4$ & $4 . \mathrm{E}-4$ & $2 . \mathrm{E}-4$ \\
\hline error & $2.725 \mathrm{E}-8$ & $1.724 \mathrm{E}-9$ & $1.016 \mathrm{E}-10$ \\
\hline order & 3.983 & 4.084 & \\
\hline \hline
\end{tabular}

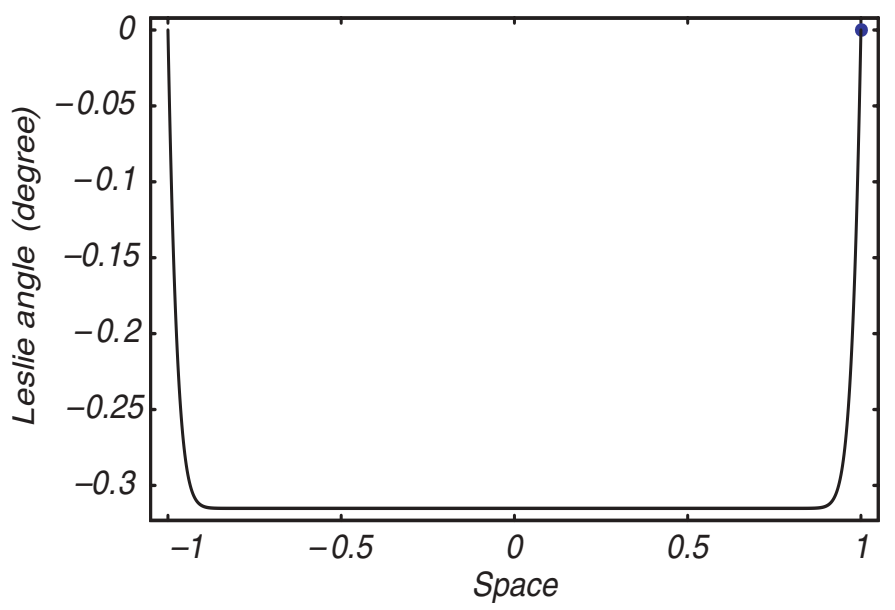

FIG. 1. Er $=500, D e=1$. Parallel anchoring. The solid line shows the result obtained using 801 uniform grid points and fixed time steps $5 \times 10^{-6}$. The dots show results using 101 adaptive grid points and adaptive time steps.

We now test the effectiveness of the adaptive grid using the same parameter values of $E r, D e$, and final time. Figure 1 shows two numerical results in two different runs. The solid line shows the Leslie alignment angle using 801 unit form grid points and fixed time step size $5 \times 10^{-6}$, while the dots show the alignment angle using 101 adaptive grid points and adaptive time steps. The final time is 0.1 . It takes 2000 steps using 801 fixed grid points and 292 steps using 101 adaptive grid points. For the adaptive grid, more than $75 \%$ of the grid points are clustered in two sharp boundary layers. Finally, we set the final time as 50 and show the adaptive time step size and grid adaptivity in Figure 2. The attracting state of the nematic polymers in the chosen regime is periodic. From Figure 2, time steps and the physical grid adapt to the numerical solutions nicely.

4. Application: Slow plate structures for strong anisotropic distortional elasticity. In this section, we apply these numerical tools to determine flow and molecular structure formation in slow Couette flow with low Ericksen numbers. This viscoelastic flow regime allows a comparison of the resolved kinetic model with analytical and numerical results on mesoscopic models derived from the kinetic system with isotropic [11] and anisotropic [2] distortional elasticity. The parameters fixed in the computations are infinitely thin rods with aspect ratio parameter $a=1$, molecular concentration $N=6$ in the quiescent nematic region, relative strength of elastic energy to kinetic energy $\alpha=2$, Reynolds numbers $\mu_{1}=0.0004, \mu_{2}=0.15, \mu_{3}=0.01$ (which are values for long rods with $r \approx 10$ ), and anisotropic elasticity parameter 

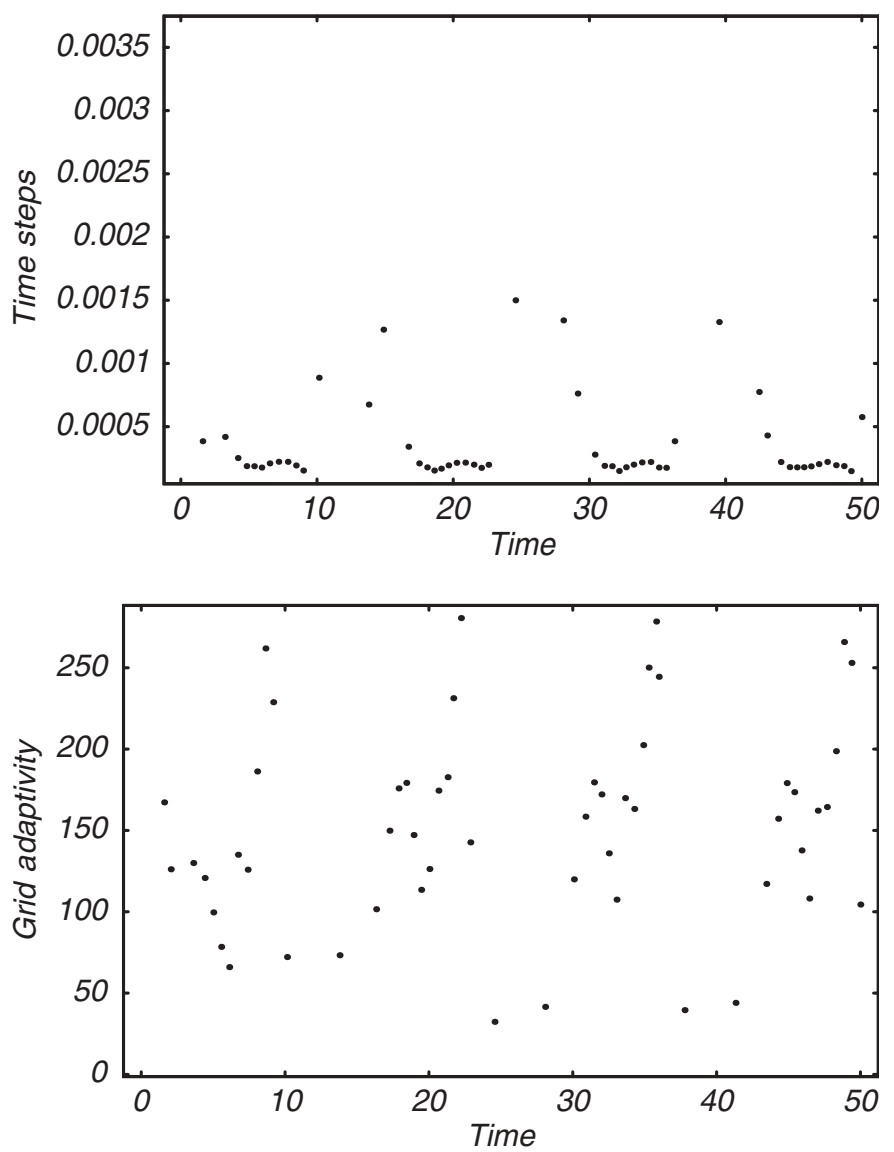

FIG. 2. The top image shows adaptive time step size in temporal integration. The bottom image shows the ratio of the maximum to minimum grid spacing versus time, which indicates the adaptivity of the grid to resolve fine features.

$\theta=0.5$, which yields distinct Frank elastic constants (see [28] for explicit values). We vary the Ericksen number $E r$ and the Deborah number De in our computations, retaining low De and low $E r$ where analytical and numerical mesoscopic results are available. All computations are limited to in-plane orientational distributions for comparison with $[2,11]$; i.e., we solve the Smoluchowski equation in the subspace in which $a_{l}^{m}=0$ for all odd superscripts $m$. In this subspace, the out-of-plane components $Q_{x z}$ and $Q_{y z}$ of the second-moment orientation tensor derived from PDF $f$ are identically zero. Therefore, one director is fixed along the vorticity axis, with two directors in the shear plane. The peak of the PDF is then associated with the major director $\mathbf{n}$, which has the form

$$
\mathbf{n}=(\cos \phi, \sin \phi, 0)
$$

where $\phi=\phi(y, t)$ is called the in-plane Leslie alignment angle. From a multiscale perspective, the upscaling from the $\operatorname{PDF} f$ to the single director $\mathbf{n}$ reveals the primary descriptive variables of kinetic and continuum theory.

Three different boundary anchoring conditions prescribed by (44) and (49) are used: parallel anchoring $\phi_{0}=0$, normal anchoring $\phi_{0}=\pi / 2$, and tilted anchoring 

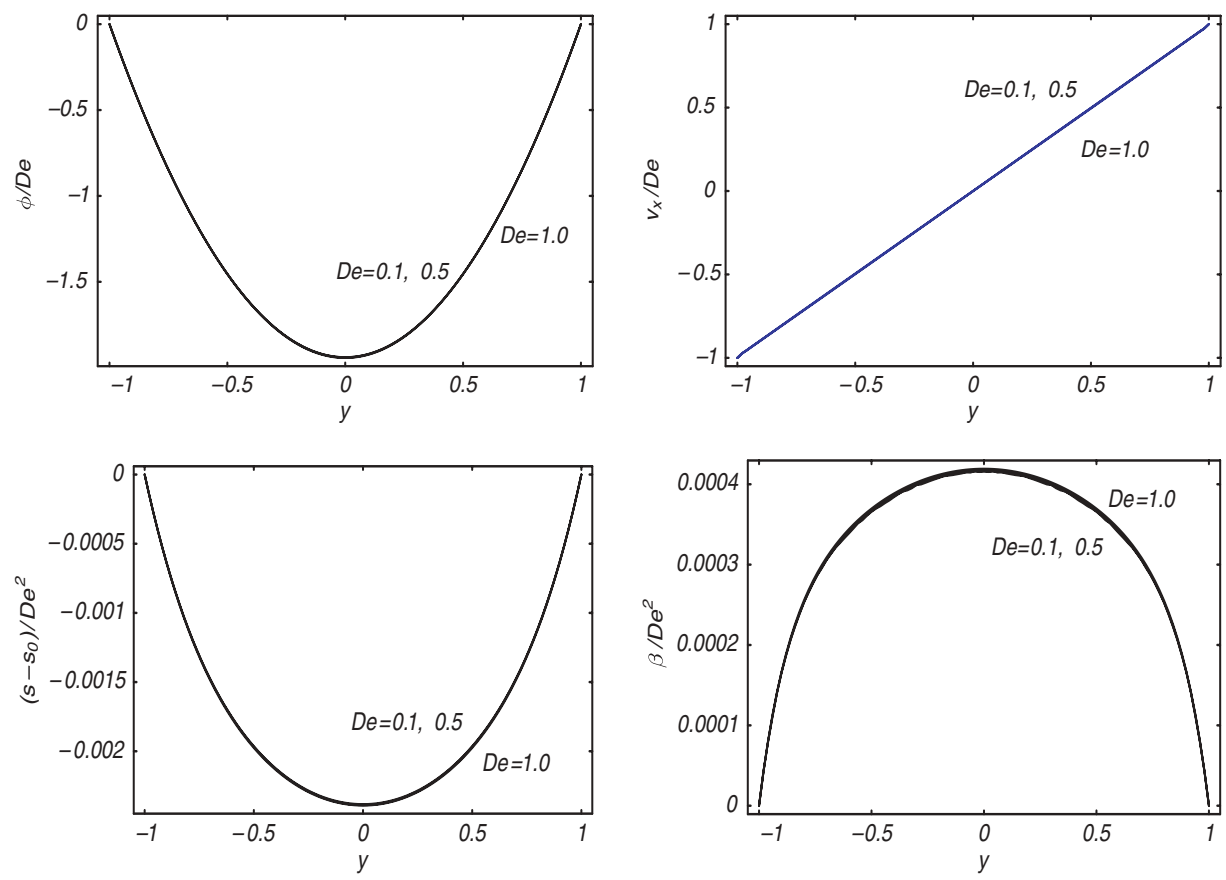

FIG. 3. Weak shear structure properties for $E r=5, D e=0.1,0.5,1.0$ with parallel anchoring $\left(\phi_{0}=0^{\circ}\right)$. Frank elastic constants are distinct $(\theta=0.5)$. In all images, the angle $\phi$ is depicted in degrees.

$\phi_{0}=\pi / 6$. Initially, the molecular distribution is in a homogeneous rest state, given by the quiescent nematic equilibrium

$$
f(\mathbf{x}, \mathbf{m}, t=0)=f_{e}(\mathbf{m}) .
$$

Figure 3 shows steady state profiles that result in parallel anchoring $\left(\phi_{0}=0\right)$ for Ericksen number $E r=5$ and three different Deborah numbers $D e=0.1,0.5,1.0$. This regime of $D e$ and $E r$ corresponds to extreme elasticity which dominates the flow feedback and is shown analytically in $[2,11]$ to yield simple steady flow profiles at leading order in De. The steady state PDF $f(\mathbf{m}, y)$ maintains essentially a uniform shape of the orientational distribution across the plate gap, conveyed by the order parameters $(s, \beta)$ which deviate negligibly from the equilibrium values $\left(s_{e q}, \beta_{e q}\right)$ imposed at the plates. This result means there is negligible "molecular elasticity" in this slow flow, high elasticity regime. The primary effect due to shear and confinement is therefore in the rotation of the peak axis (major director) of the PDF, so-called nematic elasticity, which is consistent with the Ericksen-Leslie continuum theory limit of the kinetic model. Note that the director distortions are insensitive to $D e=0.1,0.5,1.0$. These flow and molecular properties are remarkably consistent with structure scaling laws derived in $[2,11]$.

Figure 4 shows results for normal anchoring $\left(\phi_{0}=\pi / 2\right)$ for the same Ericksen number $E r=5$ and Deborah numbers $D e=0.1,0.5,1.0$. Similar to parallel anchoring, the major director alignment angle scales linearly with $D e$ with a parabolic structure shape, while the order parameters $s$ and $\beta$ experience much weaker distortions proportional to $D e^{2}$. The velocity field is again simple linear shear. For tilted 

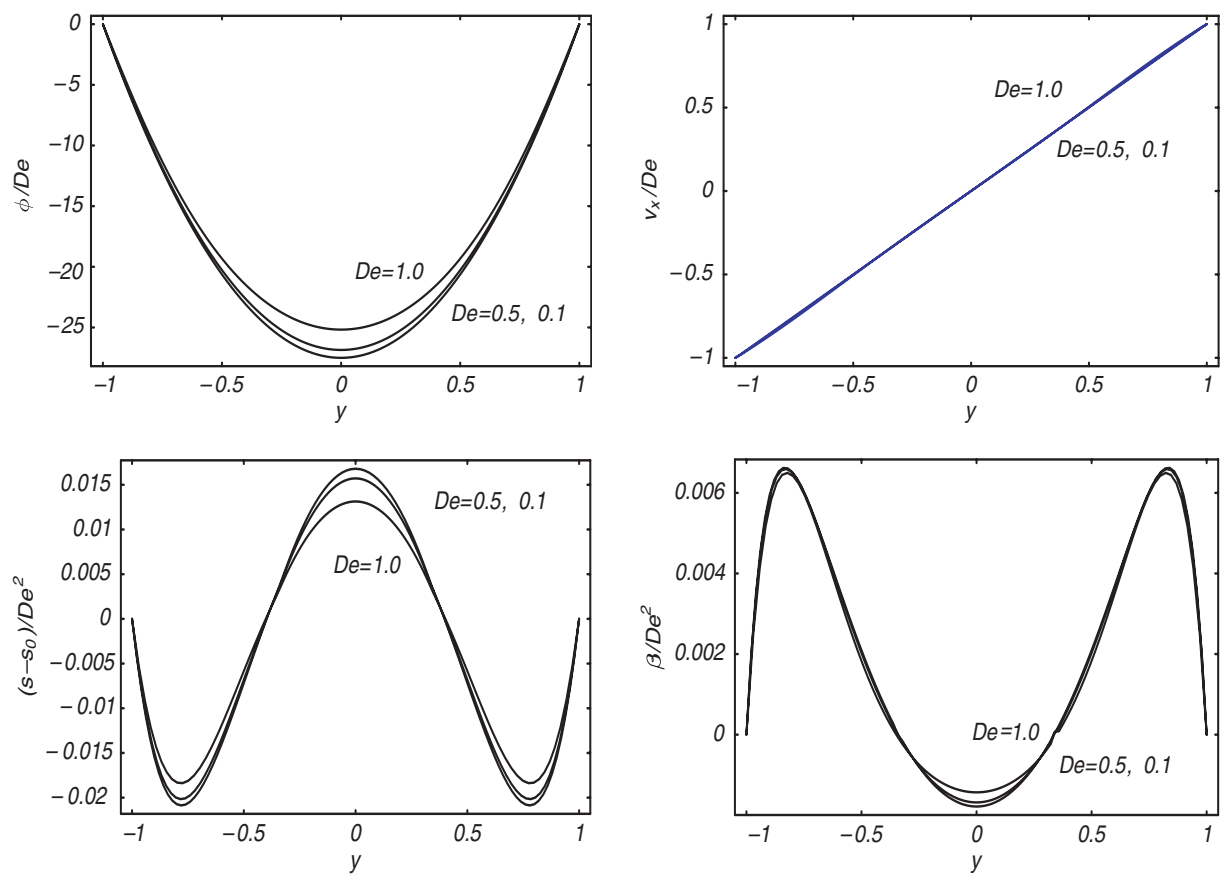

FIG. 4. Weak shear structure properties for $E r=5, D e=0.1,0.5,1.0$ with normal anchoring $\left(\phi_{0}=90^{\circ}\right)$. Frank elastic constants are distinct $(\theta=0.5)$.

anchoring, we keep $E r=5$ but lower the Deborah number to $D e=0.01,0.1,0.5$ as suggested by analytical results in $[2,11]$. Results are shown in Figure 5. Contrary to parallel and normal anchoring, the order parameters are amplified to $O(D e)$ and have the same order structure effects as the alignment angle. This means that tilted anchoring generates molecular and nematic elasticity for the same De and Er, a phenomenon predicted by mesoscopic analysis in [11,2]. Note further the first evidence of growth of nonlinear flow feedback that accompanies the amplified molecular elasticity.

All results above are for distinct Frank elastic constants with $\theta=0.5$ in the stress formula (6). For the isotropic case where all constants are equal $(\theta=0)$, the structures are nearly identical in this asymptotic regime; we show the results for normal anchoring in Figure 6. The effect of anisotropic elastic constants at higher $E r$ and $D e$ is deferred to another communication.

5. Conclusion. A numerical algorithm has been developed and benchmarked for the study of structure formation of nematic polymers in planar Couette cell experiments. The model provides a kinetic-scale resolution of the molecular orientational distribution and allows feedback between hydrodynamic and molecular fields. We show that in the dual limit of low Deborah number (slow plate) and low Ericksen number (where distortional elasticity dominates short-range excluded volume effects), mesoscopic second-moment closure models are extremely accurate predictors of steady flow molecular structure phenomena. These results give a foundation for continuation into the high Ericksen number and moderate-to-high Deborah number regime, where second-moment closure models are notoriously sensitive even in the longwave limit for imposed simple linear flow fields $[22,9,10]$. 

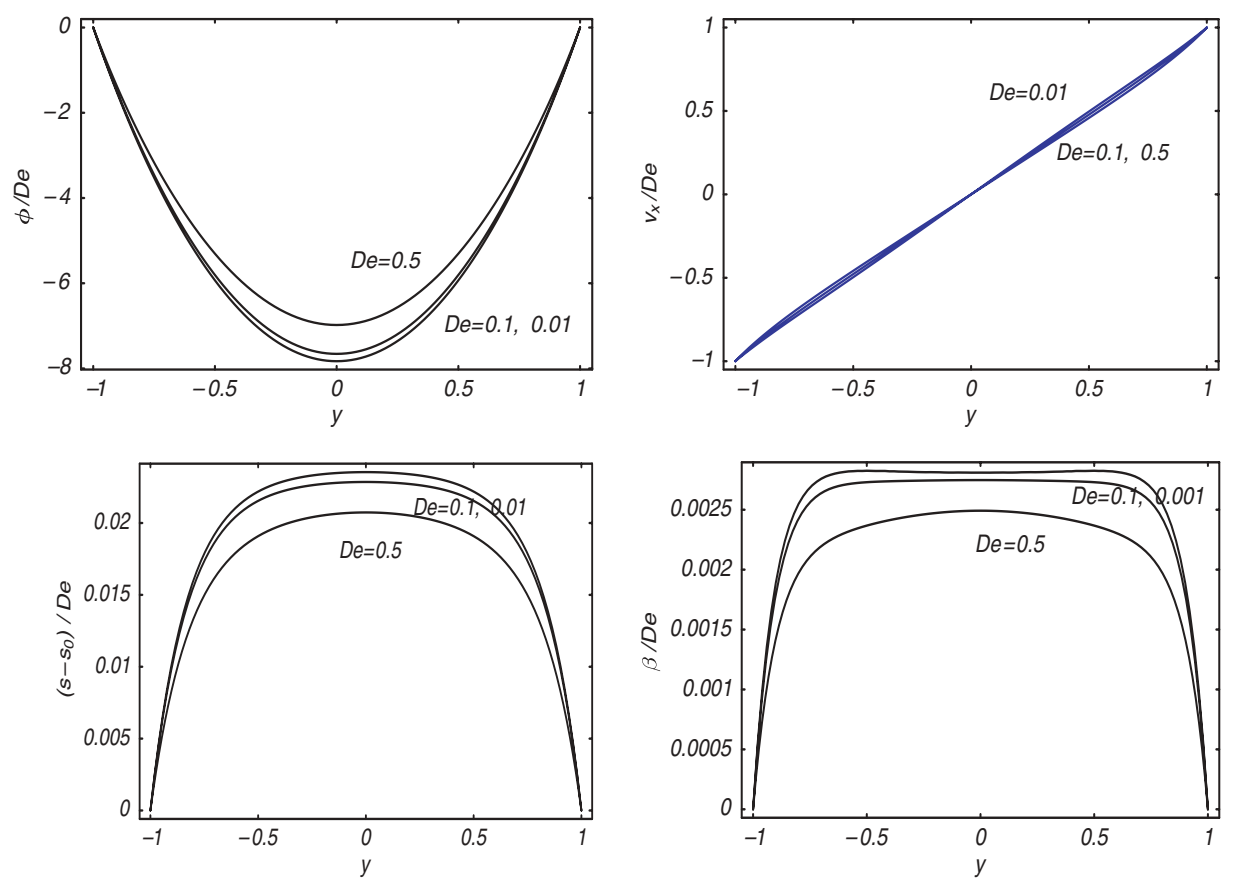

FIG. 5. Weak shear structure properties for $E r=5, D e=0.01,0.1,0.5$ with tilted anchoring $\left(\phi_{0}=30^{\circ}\right)$. Frank elastic constants are distinct $(\theta=0.5)$.
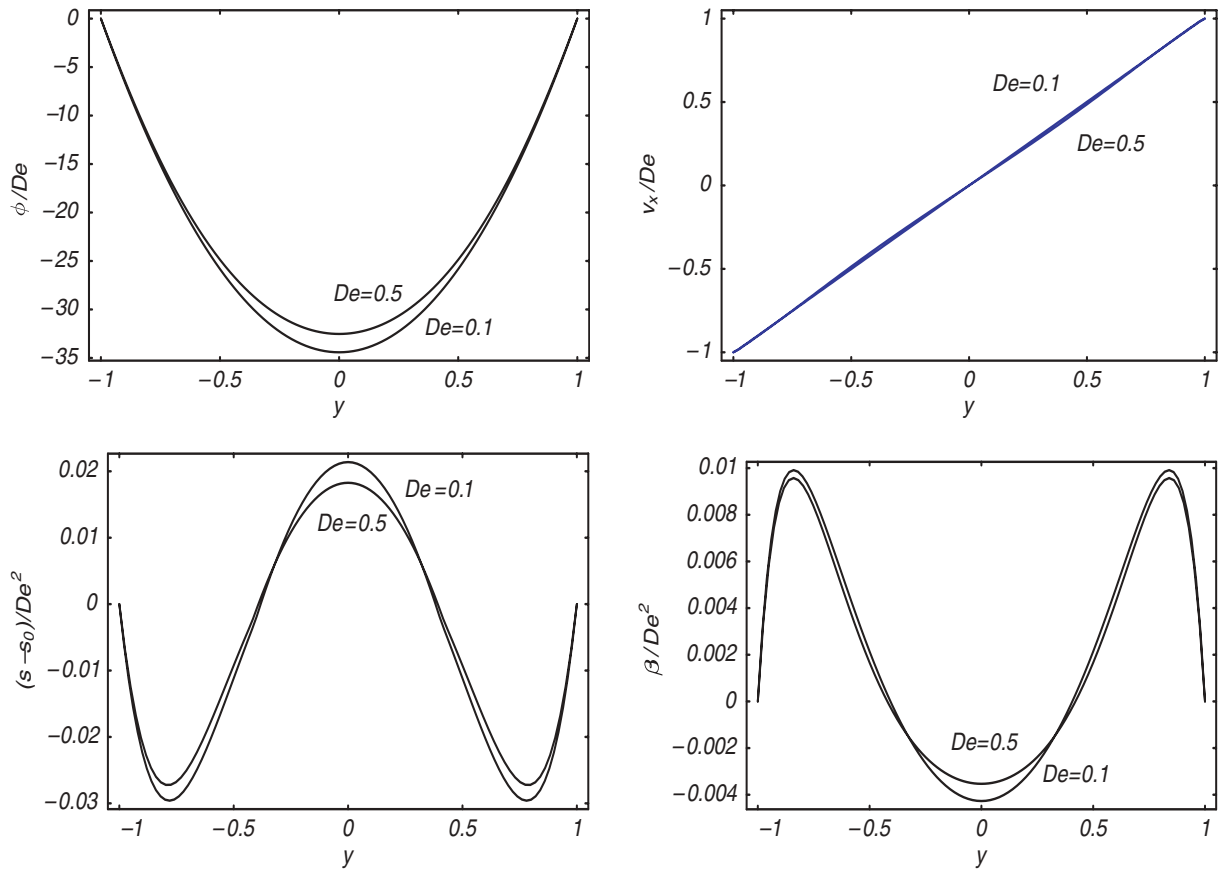

FIG. 6. Weak shear structure properties for $E r=5, D e=0.1,0.5$ with normal anchoring $\left(\phi_{0}=90^{\circ}\right)$. Frank elastic constants are all equal $(\theta=0)$. 


\section{REFERENCES}

[1] M. E. Cates and M. R. Evans, eds., Soft and Fragile Matter, Scottish Universities Summer School in Physics \& Institute of Physics Publishing, Bristol, Philadelphia, 2000.

[2] Z. Cui, M. G. Forest, And Q. WAng, On weak plane Couette and Poiseuille flows of nematic polymers, SIAM J. Appl. Math., submitted.

[3] P. G. De Gennes and J. Prost, The Physics of Liquid Crystals, Clarendon Press, Oxford, 1993.

[4] A. Dutt, L. Greengard, and V. Rokhlin, Spectral deferred correction methods for ordinary differential equations, BIT, 40 (2000), pp. 241-266.

[5] V. Faraoni, M. Grosso, S. Crescitelli, and P. L. Maffettone, The rigid-rod model for nematic polymers: An analysis of the shear flow problem, J. Rheol., 43 (1999), pp. 829843.

[6] M. G. Forest AND Q. WANG, Monodomain response of finite-aspect-ratio macromolecules in shear and related linear flows, Rheol. Acta, 42 (2003), pp. 20-46.

[7] M. G. Forest, Q. WANG, AND H. Zhou, Anchoring-Induced Structure Transitions of Nematic Polymers in Plane Couette Cells, UNC Program in Applied Mathematics preprint, 2004.

[8] M. G. Forest, R. Zhou, And Q. Wang, Symmetries of the Doi kinetic theory for nematic polymers of arbitrary aspect ratio: At rest and in linear flows, Phys. Rev. E, 66 (2002), 031712 .

[9] M. G. Forest, Q. WAng, And R. Zhou, The weak shear kinetic phase diagram for nematic polymers, Rheol. Acta, 43 (2004), pp. 17-37.

[10] M. G. Forest, Q. WAng, AND R. Zhou, The flow-phase diagram of Doi-Hess theory for sheared nematic polymers II: Finite shear rates, Rheol. Acta, 44 (2004), pp. 80-93.

[11] M. G. Forest, Q. WAng, H. Zhou, And R. Zhou, Structure scaling properties of confined nematic polymers in plane Couette cell: The weak flow limit, J. Rheol., 48 (2004), pp. $175-192$.

[12] M. G. Forest, R. Zhou, AND Q. WANG, Chaotic boundaries of nematic polymers in mixed shear and extensional flows, Phys. Rev. Lett., 93 (2004), 088301.

[13] M. G. Forest, R. Zhou, AND Q. WANG, Kinetic structure simulations of nematic polymers in plane Couette cells, II: In-plane structure transitions, Multiscale Model. Simul., submitted.

[14] R. Frank, J. Hertling, And H. Lehner, Defect correction algorithms for stiff ordinary differential equations, in Defect Correction Methods, Comput. Suppl. 5, Springer-Verlag, 1984, pp. 33-41.

[15] D. Grecov And A. D. Rey, Shear-induced textural transitions in flow-aligning liquid crystal polymers, Phys. Rev. E, 68 (2003), 061704.

[16] M. Grosso, R. Keunings, S. Crescitelli, and P. L. Maffettone, Prediction of chaotic dynamics in sheared liquid crystalline polymers, Phys. Rev. Lett., 86 (2001), pp. 31843187.

[17] E. Hairer and G. Wanner, Solving Ordinary Differential Equations II, Stiff and DifferentialAlgebraic Problems, Springer-Verlag, New York, 1996.

[18] P. Knupp And S. Steinberg, Fundamentals of Grid Generation, CRC Press, Boca Raton, FL, 1993.

[19] R. Kupfermann, M. Kawaguchi, and M. M. Denn, Emergence of structure in a model of liquid crystalline polymers with elastic coupling, J. Non-Newt. Fluid Mech., 91 (2000), pp. $255-271$.

[20] R. G. LARSON AND H. OtTINGER, The effect of molecular elasticity on out-of-plane orientations in shearing flows of liquid crystalline polymers, Macromolecules, 24 (1991), pp. 6270-6282.

[21] G. Marrucci, Tumbling regime of liquid-crystalline polymers, Macromolecules, 24 (1991), pp. $4176-4182$.

[22] G. Marrucci and F. Greco, Flow behavior of liquid crystalline polymers, in Advances in Chemical Physics, Vol. 86, I. Prigogine and S. Rice, eds., Wiley, New York, 1993, pp. 331-404.

[23] A. Messiah, Quantum Mechanics, Vol. II, North-Holland, Amsterdam, 1961.

[24] O. Radulescu, P. D. Olmsted, J. P. Decruppe, S. Lerouge, J. F. Berret, and G. Porte, Time scales in shear banding of wormlike micelles, Europhys. Lett., 62 (2003), pp. 230236.

[25] G. Sgalari, G. L. Leal, AND J. FenG, The shear flow behavior of LCPs based on a generalized Doi model with distortional elasticity, J. Non-Newt. Fluid Mech., 102 (2002), pp. 361-382. 
[26] T. Tsuji And A. D. Rey, Effect of long range order on sheared liquid crystalline polymers, Part 1: Compatibility between tumbling behavior and fixed anchoring, J. Non-Newt. Fluid Mech., 73 (1997), pp. 127-152.

[27] R. A. VAIA, Polymer nanocomposites open a new dimension for plastic and composites, The AMPTIAC Newsletter, 6 (2002), pp. 17-24.

[28] Q. WANG, A hydrodynamic theory for solutions of nonhomogeneous nematic liquid crystalline polymers of different configuration, J. Chem. Phys., 116 (2002), pp. 9120-9136. 